\title{
Análisis perceptivo de la infraestructura verde urbana en Madrid a partir del Software NVIVO.
}

\author{
Rocío Santo-Tomás Muro, Eva J. Rodríguez Romero¹ y Carlota \\ Sáenz de Tejada Granados ${ }^{2}$
}

${ }^{1}$ Departamento de Arquitectura y diseño, Escuela Politécnica Superior, Universidad San

Pablo CEU-CEU Universities, España | rociosantotomasmuro@ceu.es; rodrom@ceu.es | https://orcid.org/0000-0002-8577-7008; https://orcid.org/0000-0003-2550-3774

2 Departamento de Arquitectura y diseño, Escuela Politécnica Superior, Universidad San Pablo CEU-CEU Universities, España; Barcelona Institute for Global Health (ISGlobal)

CREAL, España | car.saenzdetejada@ceindo.ceu.es | https://orcid.org/0000-0001-87121929

Resumen: Introducción El estudio del paisaje en general, y de las infraestructuras verdes en particular, es especialmente complejo, principalmente para atender a los aspectos perceptivos que exige toda caracterización. El uso de un análisis cualitativo para el estudio de la percepción en los casos de estudio permite profundizar en los detalles y matices que surgen al analizar lo que la literatura ha denominado la visión «no experta» del paisaje Objetivos; Métodos Para este análisis de la percepción de la infraestructura verde urbana se utiliza una metodología cualitativa, apoyada en el software NVIVO. El software se usa para codificar las respuestas de una serie de entrevistas semiestructuradas que se realizan en los casos de estudio; parques metropolitanos del contorno de la ciudad de Madrid, que permita estudiar su percepción. Para la codificación, se hace un proceso de "decantación": se empieza por una codificación abierta, se pasa a una codificación axial, para terminar con una codificación selectiva, creando así una jerarquía entre los principales conceptos (denominados "nodos") que surgen a lo largo de las entrevistas Resultados Conclusiones El análisis metodológico propuesto tiene como objetivo entender la percepción de los elementos que forman parte de la red de infraestructura verde del contorno de Madrid. Los resultados obtenidos demuestran que estas técnicas de entrevista aportan resultados significativos con una muestra relativamente pequeña de entrevistas. Tras el desarrollo de esta investigación, se ha optado por el uso de esta nomenclatura: «técnica» y "cotidiana» para referirnos a las miradas al paisaje. Así, se ahonda en el sentido del lugar, la conectividad, multifuncionalidad y accesibilidad de la infraestructura verde urbana o en la imagen de la ciudad.

Palabras clave: Percepción del Paisaje; Infraestructura Verde Urbana; Madrid; NVIVO; Investigación Cualitativa.

\section{Perceptual Analysis of the Urban Green Infrastructure in Madrid Using NVIVO Software}

Abstract. The study of landscape in general, and green infrastructure in particular, is particularly complex, mainly to address the perceptual aspects required for any characterization. The use of a qualitative analysis for the study of perception in the case studies allows us to delve into the details and nuances that arise when analyzing what the literature has referred to as the "non-expert" view of the landscape Goals; Methods In order to analyse the perception of urban green infrastructure, we use a qualitative methodology, supported by NVIVO software. The software is used to code the responses of a series of semistructured interviews conducted in the case studies; metropolitan parks in the outskirts of the city of Madrid, to study their perception. For coding, a "decantation" process is used: starting with an open coding, followed by an axial coding, and ending with a selective coding, thus creating a hierarchy among the main concepts (called "nodes") that emerge during the interviews. Results (The proposed methodological analysis aims to understand the perception of the elements that are part of the green infrastructure network around Madrid. The results obtained show that these interview techniques provide significant results with a relatively smal sample of interviews. After the development of this research, we have opted for the use of this nomenclature: "technical" and "everyday" to refer to the views to the landscape. Thus, we delve into the sense of place, connectivity, multifunctionality and accessibility of urban green infrastructure or the image of the city.

Keywords: Landscape Perception; Urban Green Infrastructure; Madrid; NVIVO; Qualitative Research. 


\section{Introducción}

El estudio del paisaje en general, y de las infraestructuras verdes en particular, es especialmente complejo, principalmente para atender a los aspectos perceptivos que exige toda caracterización. Se utiliza un análisis cualitativo para el estudio de la percepción en los casos de estudio permite profundizar en los detalles y matices que surgen al analizar lo que la literatura ha denominado la visión «no experta» del paisaje (Zube et al., 1982; Larrère \& Larrère, 2009). En este trabajo se explora si el software NVIVO es una herramienta válida que permite analizar, a partir de una «mirada técnica», la «mirada cotidiana» de las personas que experimentan un lugar.

La metodología aquí presentada forma parte de una propuesta más amplia de técnicas de análisis perceptivo, desarrollada en la tesis doctoral de la primera autora (Santo-Tomás Muro, 2021), centrada en el estudio de la infraestructura verde urbana del contorno de la ciudad de Madrid, con la creación de herramientas metodológicas que puedan ser aplicadas en futuros estudios y/ proyectos sobre infraestructura verde, e incluso en otro tipo de espacios.

\subsection{La Percepción del Paisaje y los Factores Subjetivos de la Misma}

La percepción juega un papel fundamental a la hora de valorar y proteger el paisaje. Depende tanto de los elementos del mundo físico, como de la relación con otras personas y de la experiencia individual subjetiva, combinando factores sociales y culturales que dan lugar a la identidad de un lugar (Greenbie, 1982). Pese a que los espacios naturales y rurales han sido bastante estudiado en los últimos años, la percepción y valoración de los entornos urbanos complejos ha sido menos explorada.

A pesar de que algunos factores perceptivos influyeron en el diseño del paisaje, sobre todo desde el siglo XVIII, no es hasta la segunda mitad del siglo XX cuando se empezaron a estudiar de manera sistemática los atributos subjetivos del paisaje. Para ello, se crearon herramientas de valoración del paisaje que derivaron en una legislación de protección de espacios por su valor estético e impacto visual, como las «carreteras escénicas» y la protección de zonas costeras, especialmente en Reino Unido y en EEUU.

Así, se empezó a fraguar el concepto de «psicogeografía», relacionado con lo que se conoce como «psicología ambiental» (Hellpach, 1935; Hernández Ruiz y Jiménez Burillo, 1988). También se estudió la relación entre la ciudad y los habitantes desde una perspectiva perceptiva (Kevin Lynch, 1960; Gordon Cullen,1961), definiendo la base de la «teoría del lugar», que afirma que la planificación urbana, debe tener asociadas interpretaciones emotivas y significados que muestren su identidad, para poder adquirir la verdadera categoría de lugar (Cruz Pérez y Español-Echániz, 2009)

La falta de una base teórica y metodológica que aclarase los distintos puntos de vista a la hora de estudiar el paisaje llevó a definir los paradigmas principales de la percepción del paisaje (Zube et al., 1982), basados en la relación entre ser humano y naturaleza: el «paradigma del observador experto» donde el observador está entrenado y tiene nociones de arte, diseño o ecología (Burke, 1975; Duffield y Coppock, 1975), el «paradigma psicofísico», donde se defiende que la percepción depende de una serie de estímulos externos, que el observador percibe sin ser consciente (Clamp, 1976; Crystal y Brush, 1978), el «paradigma cognitivo», que busca entender el significado asociado al paisaje en función de experiencias, memorias, expectativas y condiciones socioculturales (Price, 1979; Riley, 1979) y el «paradigma experiencial», donde se considera que los valores asociados al paisaje se basan en la interacción con el mismo, en un proceso activo (Lowenthal, 1968; Duncan, 1973).

Mientras que los dos primeros paradigmas se relacionan con el contexto específico del lugar, y comparten un enfoque práctico, los últimos dos intentan entender el contexto antrópico, y, por tanto, tienen una mayor aplicación en los estudios perceptivopaisajísticos, con un enfoque en el significado y en la interacción con el entorno. 
A medida que avanzan los años se está abogando por un estudio cada vez más sistemático del paradigma experiencial y cognitivo, que refleje una relación más directa entre los espacios y la práctica del lugar.

En el sentido de la valoración estética, Lothian define el acercamiento «objetivo» y el acercamiento "subjetivo", muy relacionado con lo que también se ha denominado «análisis experto» y "análisis no experto». Mientras que el primero (generalmente relacionado con el "análisis experto") se basa en los "elementos inmutables sobre los que se puede hacer un juicio absoluto, independientemente del tiempo y del lugar" (Lothian, 1999; Pardo-García y Mérida-Rodríguez, 2017), el acercamiento subjetivo defiende la idea que el propio sentido estético del lugar depende de la interacción entre paisaje y observador (Kaplan y Kaplan, 1989). Por tanto, el primer paradigma defiende los elementos inherentes en el paisaje físico, el segundo depende de la mente del observador (Nogué, 1992).

\section{Metodología}

Se realiza el diseño de unas entrevistas semiestructuradas que después son analizadas con el software NVIVO, para más tarde relacionarlas con conceptos teóricos. Se combinan documentos escritos (transcripción de las entrevistas) con análisis de imágenes (dibujos de los entrevistados).

El estudio de casos se ha desarrollado en seis parques del contorno de la ciudad de Madrid: el Parque Lineal del Manzanares, Madrid Río, Dehesa de la Villa, el Parque Forestal de Valdebebas, el Parque Juan Carlos I y la Cuña Verde de Moratalaz, seleccionados en función de las características principales de la infraestructura verde urbana: conectividad, multifuncionalidad y accesibilidad (Mell, 2010). Además, fueron escogidos por presentar propiedades que hicieran posible su comparación de tipo morfológico, de carácter, de escala y de relación entre la ciudad y el campo. Debido al enfoque metodológico de esta comunicación, no desarrollaremos en profundidad los resultados de ninguno de los casos, sino los aspectos puramente metodológicos de la investigación en su conjunto.

\subsection{Trabajo de Campo. Toma de Datos}

El trabajo de campo incluye dos fases: una primera de observación del entorno y documentación, que da lugar a una pre-caracterización bajo el filtro de la «visión experta», y una segunda que consiste en entrevistas a personas que estén en el entorno estudiado, reflejando la «visión no experta». El fin del trabajo de campo es llegar a una evaluación de la percepción desde ambos puntos de vista.

Cada caso de estudio se visita dos veces: una vez entre semana y otra en fin de semana. Para cada visita, se recorre la zona de estudio, anotando las condiciones ambientales y las primeras impresiones del entorno, y se toman fotografías que documentan el estado del lugar. Tras esta primera fase, se procede a realizar las entrevistas. 
Para el estudio de la "visión no experta» se opta por combinar entrevistas semiestructuradas con dibujos del lugar in situ. Para las "entrevistas semiestructuradas», se plantea un guión con una serie de preguntas abiertas, a modo de ficha, con temas posibles (Fig.1), pero priorizando que el entrevistado dé su opinión y guíe la entrevista como mejor considere, pudiendo añadir preguntas durante la conversación, con nuevos temas surgidos. Esto permite cierta flexibilidad, donde los conceptos surgidos son fieles a la experiencia del lugar de los entrevistados.

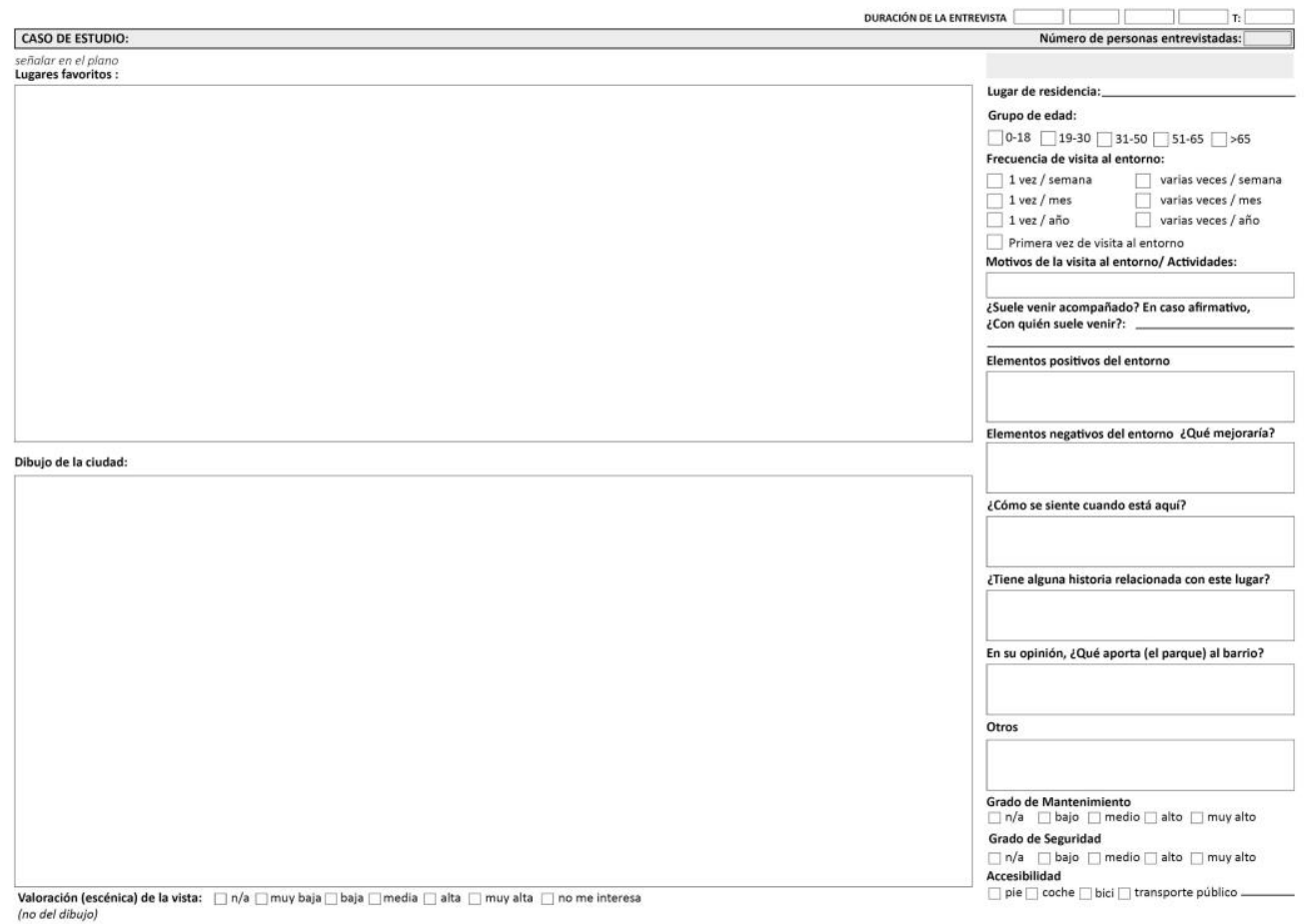

Fig. 1. Modelo de ficha para las entrevistas. Santo-Tomás Muro, R (2021).

Durante el «muestreo teórico» (Hernández Carrera, 2014), esto es, la recogida de datos, los entrevistados son seleccionados de forma fortuita, abordados directamente en los casos de estudio (zonas mirador) entre las 10:30 y las 12:30 horas de la mañana, en días con buena visibilidad y sin probabilidad de lluvia. Se realizan una media de cinco entrevistas por cada caso y día. Para la selección de los participantes se prima la mayor variedad de edades. Además, se realizan entrevistas tanto individuales como colectivas, para reflejar las diferentes experiencias en los casos de estudio.

Se llega a la "saturación teórica», cuando los datos obtenidos permiten desarrollar los objetivos de la investigación, sin que sean necesarias nuevas entrevistas, cuando ya no surgen nuevos conceptos, y empiezan a repetirse las respuestas (Mason, 2002; Valles, 2002; Hernández Carrera, 2014).

Entre las preguntas que se realizan están: nombrar elementos positivos y negativos del entorno, describir cómo se sienten en el lugar, relatar alguna anécdota en el lugar, detallar qué aporta el caso de estudio al barrio, calificar el mantenimiento y la seguridad, y señalar la forma de acceso al entorno y la frecuencia en que lo visitan.

Por último, se pide a los entrevistados que realicen un dibujo desde el mirador donde se encuentran en relación con la ciudad como única instrucción. Aunque esta parte produce en ocasiones un rechazo inicial por parte de los entrevistados, especialmente de los de mayor edad, una vez que comienzan a dibujar, suelen mostrarse más participativos. 
La calidad técnica y gráfica no tiene importancia a la hora de analizar las respuestas; el hecho de que dibujen ciertos elementos muestra a lo que otorgan mayor relevancia, demostrando una actitud activa, mientras que si realizaran una fotografía sólo se mostraría lo que se ve desde la zona, sin más filtro subjetivo por parte de cada entrevistado que el encuadre elegido.

\subsection{Análisis de Datos Cualitativos a través del Programa NVIVO}

Este análisis se realiza a partir del software NVivo 12 Plus for Windows, que permite gestionar y analizar la información recogida en el trabajo de campo. El programa permite trabajar con textos, ficheros de audio, vídeo e imágenes, a través de la o codificación del material audiovisual (Meneses, 2019). A pesar de que el programa es una herramienta útil para el almacenamiento de datos y su codificación, el investigador es el analista que interpreta y codifica la información, y no el programa (Bergin, 2011).

Una vez importados la transcripción de entrevistas y dibujos, se clasifican en función de una serie de atributos o propiedades: caso de estudio, sexo, grupo de edad, grado de seguridad, grado de mantenimiento, valoración escénica, frecuencia de la visita, número de personas entrevistadas, tipo de acceso, día de la semana y duración de la entrevista.

La codificación se realiza a partir de la creación de «nodos», es decir, categorías de análisis; temas, conceptos, ideas o experiencias que el investigador asigna a los elementos de análisis a partir de las conexiones que surgen de las narrativas de las entrevistas. A medida que se codifican estas entrevistas y dibujos se van añadiendo nuevos nodos que surgen de su propio análisis, con lo que el proceso no es puramente lineal; para cada nodo hay un proceso de "ida y vuelta" (Sabariego, 2018; Schettini \& Cortazzo, 2015). En cada archivo se marcan las palabras, frases o imágenes y se asigna uno o varios nodos en función de su significado e intencionalidad.

Así, se realiza un proceso que podría llamarse "de decantación"; se refinan cada vez más los conceptos hasta llegar a unos nodos finales que sirvan para caracterizar las entrevistas (Fig.2). Existen distintos tipos de análisis de datos para la codificación teórica, entre los que se encuentran la "codificación abierta», "codificación axial» y la "codificación selectiva» (Weil, 2017).

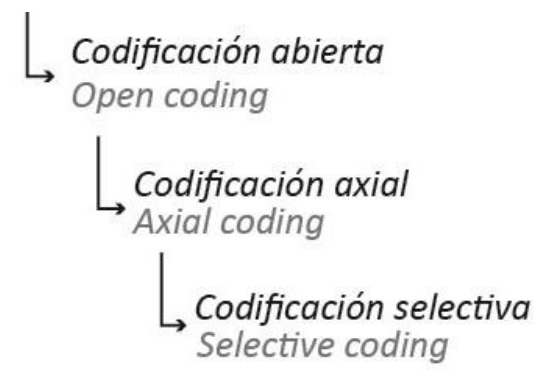

Fig. 2. Esquema del proceso de codificación. Santo-Tomás Muro, R. (2020).

El primer paso es realizar una "codificación abierta», donde se señalan frases como categorías que surgen directamente de las entrevistas. De este primer proceso se pueden extraer los temas más frecuentemente mencionados. La «codificación axial» corresponde a un primer filtro en base a relaciones de jerarquía que han surgido de la "codificación abierta", lo que supone una reorganización de las relaciones entre los primeros conceptos. Se generan categorías y subcategorías que indican las relaciones entre los nodos. La "Codificación selectiva» consiste en destacar una categoría principal entre los nodos anteriormente definidos, que se relacionan con los conceptos estudiados previamente en la teoría.

Por ejemplo, la codificación de la frase "Esto es como un mini pulmón para el barrio, incluso teniendo la carretera aquí al lado" se empezó a codificar definiendo dos nodos abiertos "pulmón del barrio" y "proximidad de las carreteras". 
Después se refinan para general nodos axiales "referencias al barrio (beneficios) e "infraestructura de trasporte". Finalmente, se clasificaron en una categoría mayor (nodos selectivos): "Contexto" e "Infraestructuras grises". Así se definen una serie de nodos para las entrevistas, y otros para los dibujos que se han realizado durante el trabajo de campo (Fig. 3).

NODOS DE LAS ENTREVISTAS

NODOS DE LOS DIBUJOS

\section{ACTIVIDADES \\ ANÉCDOTAS \\ BENEFICIOS IVU}

- Factor económico: Referencia a comercios colindantes, Mantenimiento, Precio de la vivienda

- Factor medioambiental: Naturaleza, Calidad del aire, Contaminación, Biodiversidad,

Elementos de agua

- Factor social: Menciones a la comunidad,

Salud (mental y física), Redes sociales,

Justicia Social

CONTEXTO

- Referencias al barrio: Sentido de pertenencia

- Referencias históricas: Anécdotas, Datos

históricos

- Referencias a otros espacios verdes

DISEÑO

ELEMENTOS SENSORIALES

- Auditivo

- Olfativo

- Temperatura

- Visual: Hitos, Infraestructuras Grises, Vista de la ciudad

FACTORES IVU

- Accesibilidad

- Conectividad

- Multifuncionalidad

SEGURIDAD

TIEMPO

- Días de la semana

- Estaciones

- Horas del día

\section{CONTEXTO}

- Barrio propio (dibujo de su casa)

- Barrios colindantes

- Referencias a otras IVU

ELEMENTOS ARQUITECTÓNICOS

- Cementerio

- Edificios históricos: Cornisa

- Edificios del siglo XX: CIEMAT, Edificios

de Plaza de España, Hospital 12 de octu-

bre, IFEMA, Torre Picasso, Torres KIO,

Torrespaña

- Edificios siglo XXI: Cuatro Torres Business

Area, Wanda Metropolitano

- Edificios sin identificar

ELEMENTOS DE DISEÑO

- Bancos

- Caminos, senderos

- Hitos del propio parque

- Zona de juego para niños

- Zonas para hacer ejercicio

ELEMENTOS MEDIOAMBIENTALES

- Árboles y vegetación baja

- Biodiversidad (Animales)

- Cielo, Nubes

- Contaminación

- Río, elementos con agua

- Sierra

INFRAESTRUCTURAS GRISES

- Aeropuertos, Aviones

- Artefactos eléctricos

- Carreteras, Coches

- Vías ciclistas

- Vías de tren, trenes

PERSONAS

Fig. 3. Nodos y subnodos. Santo-Tomás Muro, R (2021).

Una vez codificados los archivos con el software NVIVO, se procede a realizar «consultas», es decir, examinar las relaciones y número de referencias entre archivos, atributos y nodos. Aunque existen distintos tipos de consulta en función de las necesidades de la investigación, para este estudio se ha optado por consultas de tipo «matriz de codificación», que permiten visualizar la distribución de categorías entre los distintos archivos, cruzando datos de archivos, atributos y nodos. En el caso de la figura (Fig. 4), se muestra la cantidad de veces que el nodo axial (en este caso "elementos medioambientales") son mencionados. 


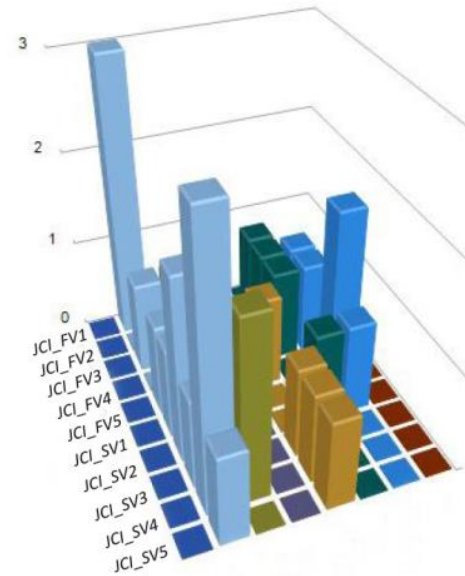

B: Biodiversidad (Animales)

A: Árboles

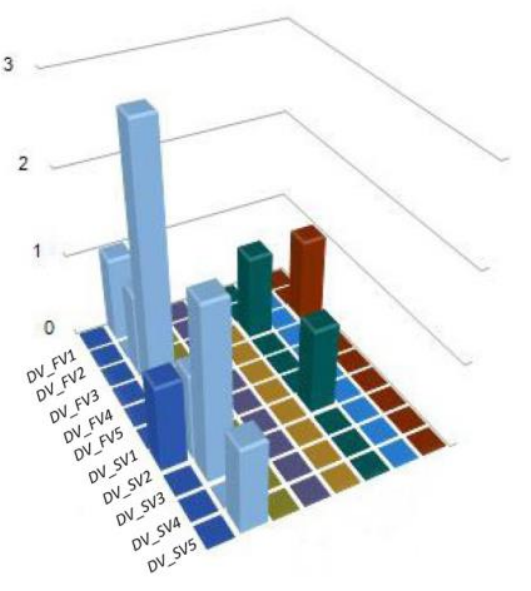

C: Cielo-Nubes

CN: Contaminación

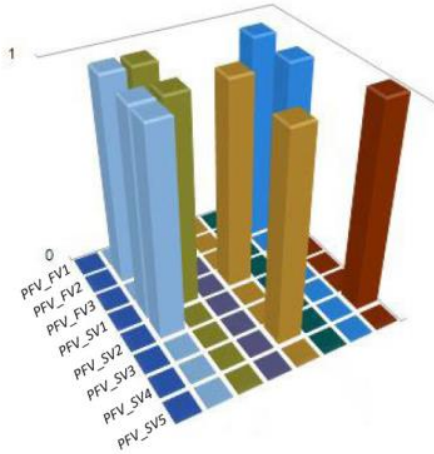

T: Topografía

Vb: Vegetación Baja

\section{LEYENDA / KEY …: $\square$ B $\square$ A $\square$ C $\square$ CN $\square$ Ag $\square$ S $\square$ T $\square$ Vb}

Fig. 4. Ejemplo de consulta tipo matriz de codificación. Santo-Tomás Muro, R (2021).

El otro tipo de consulta utilizado es el «diagrama de jerarquía» (Fig. 5), que refleja la relación porcentual de las referencias que se han dado en las entrevistas de cada uno de los nodos, lo cual permite una visión general de los temas y preocupaciones principales en cada caso.
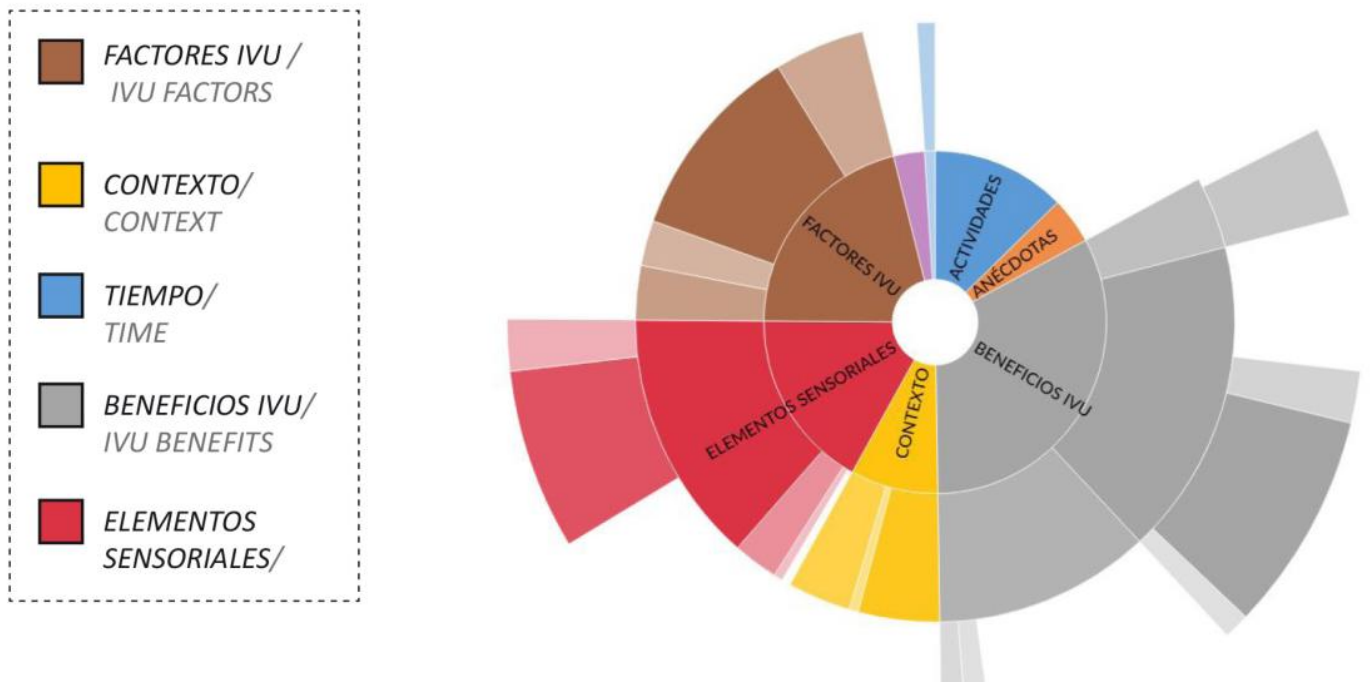

Fig. 5. Ejemplo de diagrama de jerarquía. (Santo-Tomás Muro, 2021).

\section{Resultados y Conclusiones}

El análisis metodológico propuesto tiene como objetivo entender la percepción de los elementos que forman parte de la red de infraestructura verde del contorno de Madrid, pero tiene la flexibilidad suficiente para poder aplicarse a otro tipo de casos de estudio. 
El proceso de codificación abierta, axial y selectiva ha dado lugar a categorías cada vez más orientadas al análisis. Este proceso de "decantación" de los elementos referenciados durante las entrevistas ha permitido que se apliquen relaciones de jerarquía y categorías. Los nodos más codificados a lo largo de las entrevistas fueron los "beneficios de las infraestructuras verdes", los "elementos sensoriales", y los "factores" que determinan la calidad de una infraestrura verde, como la conectividad, la multifuncionalidad o la accesibilidad.

La edad resultó ser uno de los factores que más afecta en la codificación. Por ejemplo, los entrevistados mayores de 65 años son más reacios, en general, a realizar dibujos (Fig. 6). Sin embargo, muestran un mayor apego al lugar, haciendo un mayor número de referencias a los elementos de contexto (a partir del tercer grupo de edad), por haber (la mayoría) vivido cerca del entorno durante un mayor número de años. Por el contrario, los entrevistados de menos de 30 años en general apenas mencionan el contexto.

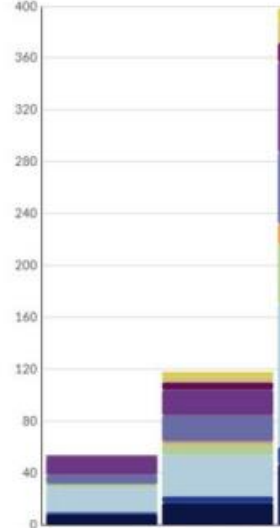

A)

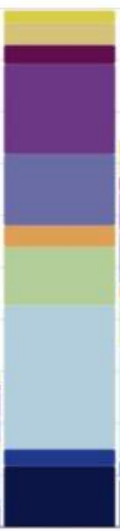

c)

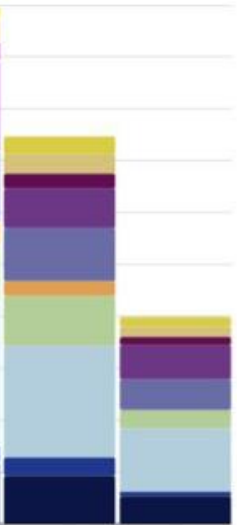

E)

D)

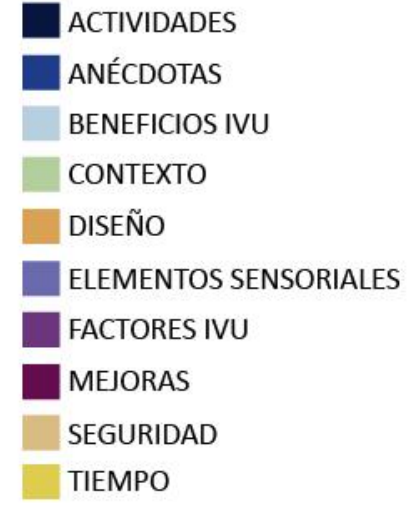

Fig. 6. Nodos codificados en función del grupo de edad: A) <18 B) 19-30 C) 31-50 D) 51-65 E) >65.

Santo-Tomás Muro, R (2021).

Para la codificación y el análisis de los datos obtenidos el software NVIVO ha resultado ser una herramienta muy ventajosa, permitiendo además del almacenamiento de las transcripciones, visualizar y cruzar los datos de forma para obtener resultados y conclusiones. Pese a la utilidad del programa, el investigador es el analista que interpreta la información, y, por tanto, la constante revisión de la codificación ha sido fundamental a la hora de interpretar los resultados.

Además, durante el trabajo de campo se ha comprobado como el dibujo resulta ser una herramienta útil a la hora de estudiar la experiencia y la mirada cotidiana de los espacios. Los dibujos actúan como una síntesis de los elementos que conforman la imagen urbana en relación con el entorno verde donde se encuentran, reflejo de dónde y cómo fijan la mirada.

Otro beneficio importante del empleo de estos métodos es que entrañan un número modesto de recursos materiales, por lo que su aplicación resulta bastante económica en tiempo y recursos. Los resultados obtenidos demuestran que estas técnicas de entrevista aportan resultados significativos con una muestra relativamente pequeña de entrevistas. Esto representa una ventaja, ya que puede utilizarse de manera ágil durante el proceso de diseño de nuevas intervenciones en la red de infraestructura verde urbana en el entorno urbano y periurbano, incluyéndose dentro del conjunto de herramientas de investigadores y profesionales.

La realización de las entrevistas en los mismos casos de estudio ha permitido experimentar el propio lugar, de modo que los entrevistados tienen reciente la experiencia del lugar, sin tener que imaginársela. Además, como parece evidente, la selección de participantes es mucho más fácil; no tienen que desplazarse a otro lugar para realizar el estudio. 
El hecho de tener un guión predeterminado (aunque flexible) permite que preguntas como "tipos de actividades realizadas en la zona" formen siempre parte de la conversación y, por lo tanto, aparezcan como temas altamente referenciados durante las entrevistas. Generalmente, las últimas preguntas se contestaban con un mayor grado de fluidez, al haberse establecido un grado de confianza entre investigador y participante con las preguntas anteriores, mientras que las primeras preguntas se contestaban de manera más mecánica, con frases cortas o monosílabos.

Aunque durante la entrevista no se limitaba el tiempo, es cierto que los entrevistados preferían contestar relativamente rápido a las preguntas (entre 6 y 10 minutos), lo que ha dado como resultado entrevistas dinámicas a modo de conversación, lo que no ha impedido que se trataran numerosos temas. En general, los entrevistados que ya se encontraban en el lugar mientras se realizaban otras entrevistas se mostraban más proclives a realizar después una entrevista, ya que comprobaban que no les llevaría demasiado tiempo. Aún así, es cierto que tanto entre semana, como en las entrevistas donde las personas eran mayores de 65 años, la duración de las conversaciones ha sido mayor.

Se han podido analizar aspectos de la experiencia del lugar como resultado del trabajo de campo en los casos de estudio. El investigador actúa como «viajero que acompaña» a los entrevistados en sus narrativas (Kvale, 2011), recorriendo el paisaje y entablando conversaciones con las personas que se encuentra.

En este sentido, el uso de NVIVO resulta especialmente beneficioso a la hora de estudiar conceptos tan subjetivos como la experiencia del lugar y la percepción del paisaje, donde resulta necesario adoptar herrmientas cualitativas que permitan su estudio sistemático. Así, se ha podido reflexionar acerca de la experiencia cotidiana del lugar, el sentido de pertenencia y apego en relación con la infraestructura verde urbana, su papel como umbrales urbanos, la conectividad, multifuncionalidad y accesibilidad de los casos de estudio, la imagen de la ciudad o la relación entre bienestar e infraestructura verde urbana.

Aunque durante el proceso de investigación se han encontrado algunas limitaciones, fruto de la necesidad de acotar la muestra, algunas de las mismas puedes servir como semilla o acicate para nuevas líneas de investigación. Por ejemplo. Pese a visitar cada caso de estudio dos veces, para comparar las diferencias entre semana y en fin de semana, en futuras investigaciones, se podrían repetir también las entrevistas en las distintas estaciones del año, para poder comparar resultados. Lo mismo es aplicable a distintas horas a lo largo del día (mañana/tarde/noche). Una muestra más grande permitiría un estudio perceptivo más pormenorizado de cada uno de los casos.

Gracias a la flexibilidad de los métodos aquí desarrollados, la metodología y foco se podría extrapolar a otros casos y ciudades, contribuyendo a una caracterización de sus elementos verdes, con el potencial de ayudar también en la generación de nuevas estrategias frente a la infraestructura verde urbana. En términos metodológicos, también sería interesante complementar con nuevas entrevistas que incorporen el uso de la fotografía realizadas por los propios entrevistados, como nueva capa en el estudio de la mirada.

Tras el desarrollo de esta investigación, se ha optado por el uso de esta nomenclatura: «técnica» $y$ «cotidiana», para huir de la afirmación de que hay una mirada "experta» $y$ «no experta», pese a que estos fueron los términos que se emplearon en la definición de la metodología, por ser los habituales en la literatura. Realmente cabe preguntarse quiénes son los verdaderos «expertos» del lugar; si los que tienen una mirada analítica basada en conocimientos técnicos sobre el mismo ( $p$.ej. conocer la historia del lugar, estar al tanto de las condiciones urbanas, etc.) o si son los que hacen del paisaje, en general, y de los casos de estudio que nos atañen en esta investigación, en particular, parte de su día a día. 


\section{Agradecimientos}

Esta comunicación forma parte del Proyecto "El paisaje periurbano de Madrid: visiones desde la memoria hacia la nueva ciudad" (junio 2020 - mayo 2023), referencia PID2019110693RB-100), del Ministerio de Ciencia e Innovación, y la Agencia Estatal de Investigación (DOI: 10.13039/501100011033), a la cual agradecemos su financiación. Asimismo, agradecemos la Ayuda de Formación de Personal Investigador de la Fundación Universitaria San Pablo-CEU (octubre 2013 - septiembre 2020) de la primera autora de la comunicación.

\section{Referencias}

Autora (2021). La infraestructura verde urbana en Madrid. Técnicas de análisis perceptivo del paisaje en el contorno de la ciudad. Universidad San Pablo CEU.

Bergin, M. (2011). NVivo 8 and consistency in data analysis: reflecting on the use of a qualitative data analysis program. Nurse researcher, 18(3), 6-12. https://doi. org/10.7748/nr2011.04.18.3.6.c8457

Burke, R. E. (1975). National Forest Visual Management-A Blend of Landscape and Timber Management. Journal of Forestry, 73(12), 767-770. https://doi.org/10.1093/ jof/73.12.767

Clamp, P. (1976). Evaluating English landscapes -- some recent developments. Environment and Planning, 8(1), 79-92.

Cruz Pérez, L., \& Español-Echániz, I. (2009). El paisaje. De la percepción a la gestión. Ed. Liteam.

Crystal, J. H., \& Brush, R. O. (1978). Measuring scenic quality at the urban fringe. Landscape Research, 3(3), 9-14. https://doi.org/10.1080/01426397808705879

Cullen, G. (1961). The Concise Townscape. Van Nostrand Reinhold Company. https://doi.org/10.1017/ CBO9781107415324.004

Duffield, B. S., \& Coppock, J. T. (1975). The Delineation of Recreational Landscapes: The Role of a Computer-Based Information System. Transactions of the Institute of British Geographers, (66), 141-148. https://doi.org/10.2307/621629

Duncan, J. S. (1973). Landscape Taste as a Symbol of Group Identity: A Westchester County Village. Geographical Review, 63(3), 334-355. https:// doi.org/10.2307/213943

Greenbie, B. B (1982). The landscape of social symbols. Landscape Research, 7 (3) 2-6. https://doi. org/10.1080/01426398208706035

Hellpach, W. (1937). Geopsyche. Die menschen seele unterm einfluss von wetter und klima, boden und landschaft. Vierte auflage. The Journal of Nervous and Mental Disease. https://doi.org/10.1097/00005053-193708000- 00054

Hernández Carrera, R. (2014). La investigación cualitativa a través de entrevistas: Su análisis mediante la teoría fundamentada. Cuestiones pedagógicas: Revista de ciencias de la educación, 23(23), 187-210.

Hernández Ruiz, B., \& Jiménez Burillo, F. (1988). La investigación en la Psicología Ambiental en España. En Comportamiento y medio ambiente: la psicología ambiental en España (1.a ed., pp. 31-50). Consejería de Política Territorial.

Kaplan, R., \& Kaplan, S. (1989). The Experience of Nature: A psychological Perspective. Cambridge University Press.

Kvale, S. (2011). Las entrevistas en investigación cualitativa. Colección: Investigación Cualitativa. Ediciones Morata.

Larrère, C., \& Larrère, R. (2009). Du bon usage de la nature pour une philosophie de l'environnement. Champs Essais.

Lothian, A. (1999). Landscape and the philosophy of aesthetics: is landscape quality inherent in the landscape or in the eye of the beholder? Landscape and Urban Planning, 44(4), 177-198. https://doi.org/10.1016/S0169- 2046(99)00019-5 
Lowenthal, D. (1968). The American Scene. Geographical Review, 58(1), 61-88. https://doi.org/10.2307/212832

Lynch, K. (1960). Image of the City. Image of the City. The MIT Press.

Mason, J. (2002). Qualitative Researching. Sage Publications.

Meneses, C. (2019). Apuntes para trabajar con NVivo 12 Plus.

Mell, I. C. (2010) Green infrastructure: concepts, perceptions and its use in spacial planning. Newcastle University

Nogué, J. (1992). Turismo, percepción del paisaje y planificación del territorio. Estudios turísticos, $115,45-54$.

Pardo-García, S., \& Mérida-Rodríguez, M. (2017). Measurement of visual parameters of landscape using projections of photographs in GIS. Computers, Environment, 61, 56-65. https://doi.org/10.1016/j. compenvurbsys.2016.09.005

Price, C. (1979). Subjectivity and Objectivity in Landscape Evaluation. Environment and Planning A: Economy and Space, 8(7), 829-838. https://doi.org/10.1068/a080829

Riley, R. (1979). Reflections on the landscape memory. Landscape, 23(2), 11-18.

Sabariego, M. (2018). Análisis de datos cualitativos a través del programa NVivo 11 PRO Dosier 1. Tutorial del programa.

Santo-Tomás Muro, R. (2021) La infraestructura verde urbana en Madrid. Técnicas de análisis perceptivo del paisaje en el contorno de la ciudad. Universidad CEU San Pablo y Universidad CEU Cardenal Herrera.

Schettini, P., \& Cortazzo, I. (2015). Análisis de datos cualitativos en Investigación social. Procedimientos y herramientas para la interpretación cualitativa. (Universidad de la Plata, Ed.).

Valles, M. S. (2002). Entrevistas cualitativas. Cuadernos metodológicos. https://doi.org/004020239

Weil, J. (2017). Research Design in Aging and Social Gerontology: Quantitative, Qualitative, and Mixed Methods. (Routledge, Ed.).

Zube, E. H., Sell, J. L., \& Taylor, J. G. (1982). Landscape perception: Research, application and theory. Landscape Planning. https://doi.org/10.1016/0304-3924(82)90009-0 